\title{
New Approaches for the Implementation of Minimally Invasive Microelectrode Arrays Designed for Brain-Machine Interfaces
}

\author{
S. Martel ${ }^{1,2}, \mathrm{~T}$. Fofonoff ${ }^{2}$ \\ 'NanoRobotics Laboratory, Department of Computer Engineering, École Polytechnique de Montréal (EPM), \\ Campus of the University of Montréal, Montréal (Québec) Canada \\ ${ }^{2}$ BioInstrumentation Laboratory, Department of Mechanical Engineering, Massachusetis Institute of Technology (MIT), \\ Cambridge, MA, USA \\ Invited Paper \\ Correspondence: sylvain.martel@polymtl.ca
}

\begin{abstract}
Microelectrode arrays are likely to play an important role in the implementation of brain-machine interfaces. One of the major obstacles is to make existing microelectrode arrays much less invasive. In this aim, novel approaches for the implementation of these microelectrode arrays will be required. This paper suggests the use structurally bi-stable electrodes based on deployable microstructures which minimize the overall dimensions during insertion into the cortex and then deploy to increase the recording surface and lower the electrical impedance. Other design alternatives and issues such as new methods of implantation, tracking, and additional features that could make microelectrodes less invasive are also briefly described.
\end{abstract}

Keywords-Microelectrode, minimally invasive, bi-stable, size reduction, deployable structures, methods of implantation

\section{INTRODUCTION}

A brain-machine interface can be defined as an interface in which the brain accepts and controls a mechanical device as a natural part of its representation of the body [1]. A present goal of brain-machine interfaces is to provide a way for people with damaged sensory/motor functions to use their brain to control artificial devices and restore lost capabilities. Future goals could potentially include opportunities to augment brain functions, for instance by providing innovative ways to interface the brain to computer-based systems and machines.

Recent advances in neurophysiology indicate that the idea of brain-machine interfaces may soon become a reality [2]. For instance, recording and interpreting the activities of group of neurons with high temporal accuracy and at specific locations in the motor cortex is presently feasible. A major breakthrough in the development of brain-machine interfaces occurred when scientists were able to transmit a monkey's brain signals over the Internet in real time in order to control a robot arm 600 miles away [3]. Although noninvasive sensors such as multi-channel electroencephalograms (EEG) have shown some potential for future brain-machine interfaces, they currently lack the spatial resolution needed for such applications. In order to provide sufficient spatial resolution for reliable and accurate interpretation of neuronal activity, current research in this field relies primarily on arrays of microelectrodes embedded into the brain. The major limitation of these microelectrode arrays, and the main reason that prevents them from being used as potential brain-machine interfaces, is that all versions produced so far are highly invasive.

Existing arrays of microelectrodes [4] are based on three main approaches. A first approach relies on an array of microelectrodes machined from a single piece of material. A second approach stacks 2D silicon-based microelectrodes and electronics created using lithography to form a 3D array. Finally, in the simplest form, individual wires or bundles of wires are used to form the array. In all of these approaches, the size of the microelectrodes is the same during the initial insertion into the motor cortex as once fully implanted into the brain. All of these approaches may result in substantial damage to the brain tissue, leading to higher risks of complications during and after surgery. Therefore, a new approach providing similar electrical specifications while reducing the cross-sectional area of the implanted electrodes may prove to be a key element in the future implementation of brain-machine interfaces and would alleviate the limitation of current approaches.

\section{Fundamental ReQuirements}

One of the key characteristics for proper recording in the motor cortex is the electrical impedance of each microelectrode. The recording surface of each microelectrode must be large enough to obtain a sufficiently low electrical impedance for proper recordings. On the other hand, lowering the electrical impedance too much may result in lowering the signal-to-noise ratio (SNR) as well as in recording the neural activity in the vicinity of neighboring microelectrodes.

In practice, other issues that are not well understood also affect the quality of recordings. It has been speculated that brain tissue reaction to the implanted array will also impact the recording parameters of the microelectrodes. One way to reduce this reaction may be to decrease the trauma to the brain during electrode insertion. A reduction in tissue damage can be achieved not only by reducing the size of the inserted object, but also by reducing the speed at which the microelectrode is lowered into the brain, since past studies have shown that the amount of damage to brain tissue is 
strongly related to the speed at which the electrode is lowered during insertion [4].

An initial strategy to make the microelectrode less invasive would be to decrease the overall physical size of the microelectrode itself. A first step toward this end is to reduce the recording surface of each microelectrode. One possibility is the development of a new biocompatible material allowing enhanced ion transfer and hence a lower impedance for a given recording surface area. Unfortunately, it is unclear whether such a material may be developed in the relatively near future. Presently, nichrome, stainless steel, and tungsten are being used, but platinum and iridium are the materials of choice in many arrays. Past studies, including our own experience in the fabrication of microelectrode arrays [5]-[7], have shown that an electrical impedance of approximately $100 \mathrm{k} \Omega$ at $1 \mathrm{kHz}$ gives the best results for recording groups of neurons in the motor cortex. Based on such data, previous fabrication of recording microelectrodes using Platinum or Iridium resulted in recording tips of approximately $50 \mu \mathrm{m}$ in length and $40 \mu \mathrm{m}$ in diameter, decreasing to approximately $15 \mu \mathrm{m}$ to $20 \mu \mathrm{m}$ at the end of the tip [8]. Such volume, considering the recording tip alone and the relatively large number of recording sites required for accurate interpretation of the brain activity, prevents the implementation of a minimally invasive version of a brain-machine interface.

Because individual wires typically used for recording have diameters of not less than $25 \mu \mathrm{m}$, as smaller-diameter wires have difficulty breaking through the dura and can often be sidetracked when lowered into the brain, smallerdiameter microelectrodes as suggested here will also need a different and innovative method of insertion.

\section{Proposed New CONCEPTS For Size Reduction DURING INSERTION}

The proposed strategy for achieving a minimally invasive device through a reduction in the overall size of the multi-microelectrode array has three aspects. First, the cross-sectional area of the array is reduced during implantation into the brain by using bi-stable structures. Second, by eliminating the insulated structural part of the microelectrodes which is present in existing microelectrode devices used for brain studies and which contributes in great part to making the system much more invasive. The third aspect involves further decreasing the overall recording surface to attain an acceptable level of electrical impedance (and an acceptable level of artifact errors caused by the limitation of input impedance of the front-end electronics) by locating each tip closer to a target group of neurons with the help of tracking techniques,. A last technique may be considered to enhance the system by embedding neurotrophic growth factors to lower further the required dimensions of the recording surface.

\section{A. Size Reduction of the Recording Tips by Bi-Stable Shapes}

To fulfill the requirements for minimally invasive brainmachine interfaces, the dimensions of the microelectrodes must be decreased further and as such, a first new concept is proposed. The new concept is based on the development of structurally bi-stable microelectrodes, i.e. microelectrodes designed to take two volumetric stable states at different time frames, in other words, with a smaller overall volume during insertion into the brain and a larger volume to lower the electrical impedance to an appropriate value once implanted into the cortex. The three main methodologies that have been identified to implement such structurally bistable recording tips are referred to here as: deployable recording tips, molecular volumetric change, and structural growth.

Presently, in all microelectrodes only the surface of the recording tip is used and the space occupied by the inner cross-section of the tip (unexposed material between the recording surfaces at the tip) is useless for recording. It is therefore possible to decrease this relatively large volume by initially folding small sections of the overall recording surface (recording faces or membranes) in a manner that will reduce significantly the overall volume of the recording tip during insertion (first shape), and then by deploying the recording membranes once embedded into the cortex (second shape) to lower the electrical impedance appropriately. Deployable structures such as antennas have been studied for various applications and in particular for space applications where volume is a constraint during takeoff. For instance, recent studies [9], [10] have been done on a new concept for multi-purpose deployable membrane reflectors known as collapsible rib-tensioned surface (CRTS) [11]. A new family of deployable biomimetic membrane structures, referred to as leaf-folding patterns [12], appear very promising for such types of applications. Although one could envision a miniature implementation of these structures to be deployed into the brain, the higher density of the tissue as opposed to the vacuum in space prevents their use for brain-machine interfaces. Nonetheless, it is possible to modify such structures in such a way that the pressure from the tissue on the recording surfaces during deployment would be decreased sufficiently to allow them to be fully deployed. Although many alternative designs exist, one proposed basic example is depicted in Fig. 1 where deployable recording spikes are used.

As shown in Fig. 1, the recording spikes remain folded during insertion (shown by the downward arrow in the left diagram). Although the folded structures of the microelectrode could be initially glued with cyanoacrylate, hot sucrose, or dextrose, or could be previously dipped in melted carbowax, all of which would dissolve over a period of time, the resulting stiffness of these deployable structures would probably not be sufficient to cope with the density of the surrounding brain tissue. On the other hand, by pulling 
on the tethered electrical wire, one could exert a force with sufficient amplitude in the reverse direction, allowing recording spikes to deploy (right diagram of Fig. 1), hence increasing the exposed recording surface.

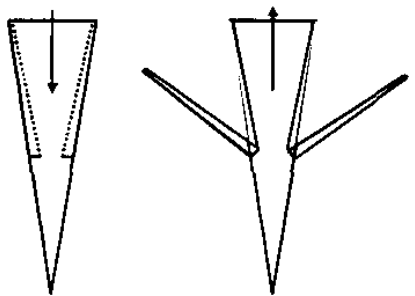

Fig. 1 - Recording tip with deployable recording spikes

Volume reduction in implantation can also be achieved through a change in the molecular structures of the material. Because of the relatively high actuation force per surface area required for deployment in the brain tissue, piezoceramics and giant magnetostrictive materials such as Terfenol-D are two potential candidates for such an application. Unfortunately, the change in volume of these materials, approximately $0.2 \%$, is too low to make an observable difference. Hence, molecular volumetric change for the purpose of decreasing the overall size of the recording tip for minimally invasive implantation does not appear to be a viable option at the present time.

Structural growth is concerned with the implantation of a high-density material compound or catalyst into the brain and growing lower-density branches or similar structures from the same compound once localized into the brain. This approach is extremely unlikely to succeed and impractical at the present time due to the lack of possible biocompatible materials used as catalysts with adequate ion transfer capabilities, coupled with the constraints imposed by the brain itself in using techniques based on growing agents, techniques such as chemical vapor deposition, or other alternatives.

\section{B. Elimination of the Insulated Structures}

In the motor cortex, the recording tip is typically positioned at a depth of approximately $1 \mathrm{~mm}$ into the brain tissue immediately below the dura. Knowing that the length of the recording tips in conventional microelectrodes is approximately $50 \mu \mathrm{m}$, it results that the insulated remaining structure between the recording tip and the dura has a length in the order of $950 \mu \mathrm{m}$, being approximately 19 times the length of a conventional recording tip. Although folding techniques for the insulated structure of the microelectrode may be envisioned at the cost of further complexity, a better approach, considering the fact that this section of the electrode has no use for recording purpose, is to eliminate it entirely. By doing so, traditional methods of pushing the microelectrode through the brain tissue during implantation are no longer viable. Therefore, the implantation force must act directly on the recording tip through the brain tissue and/or the recording tip itself must generate such a force.

\section{METHODS OF IMPLANTATION}

\section{A. Shooting}

For microelectrode arrays based on individual recording tips only, the simplest method of implantation could be to fire the tips (or one tip at a time) from a special gun using a velocity adjusted to the depth of penetration with regard to the density of the brain tissue. Then once implanted, a tiny insulated electrical.wire connecting to each recording tip could be pulled from outside the brain to create the reciprocal force needed for the deployment of the recording spikes as depicted in the right diagram of Fig. 1. Unfortunately, this proposed method has some drawbacks. First, the speed of insertion of the recording tips would be rather high, which could lead to substantial damage to the brain as mentioned previously. Second, the method does not allow for the tracking of the neurons. And third, it is unclear that very precise target localization of the recording tips could easily be achieved.

\section{B. Induced Force}

Another proposed method consists of inserting the folded recording tips by inducing sufficient force on them in order to break through the brain tissue. One possibility for generating such a force is by the use of magnetic gradients. If the recording tips and the attached membranes are made of a ferromagnetic material (e.g. stainless, cobalt, chrome, or carbon steel) coated with platinum or a similar biocompatible conductive material, then it is possible to generate sufficient magnetic gradient forces to move the tips through the tissue. Past studies have demonstrated devices that can drive through muscle tissue at a rate up to $1 \mathrm{~mm} / \mathrm{s}$. These actuators, with sizes of about $10 \mathrm{~mm} \times 2 \mathrm{~mm}$, developed at Tohoku University, are driven by a rotating magnetic field generated by two vertical coils of wire with alternating current passing through the coils, forcing a $\mathrm{NdFeB}$ permanent magnets in the screw-like actuator system to rotate at a speed proportional to the frequency of the alternating current. This method of induced force by magnetic gradients has the disadvantage that it requires high intensity fields that are not necessarily suitable to a clinical environment. Furthermore, although slow motion in all directions could potentially be feasible, making tracking of neurons very effective for at least one recording tip, the same motion would unfortunately apply to all recording tips simultaneously, reducing the global effectiveness in tracking neuronal activities for most recording tips, even if applying advanced tracking algorithms. Furthermore, it is not clear whether, in keeping the fields within acceptable safety levels, it would be possible to move tips with much smaller 
dimensions through the tissue. Further calculations and experimentations are required to validate such an approach.

\section{Embedded Micro-Actuation}

Embedding micro-actuation in each recording tip has the advantage of independent control and localization when more than one tip is implanted. Due to size constraints, polymer-based micro-actuators and micro-actuators based on magnetostriction, piezo effects, electrostatic actuation, or thermal expansion may be some of the most promising methods of actuation. Possible polymer-based actuators include conjugated polymers such as polypyrrole and a commercial perfluorosulfonic acid polymer known as Nafion. These polymer-based actuators are of particular interest here since they can change volume to deliver significant stresses and strains when electrically activated with relatively low voltage amplitudes, a real advantage especially if the miniaturization of electronics embedded into the brain becomes an issue. The relatively low resonant frequency of these actuators (although compensated by their relatively large motion amplitudes) is not of real concern for this application. However, in the case of polypyrrole, an electrolyte solution is needed as an ion source or sink to activate the material, preventing its use in the brain tissue unless the electrolyte solution is encapsulated within the actuator itself. Although this may be feasible, this would increase the implementation complexity and it is unlikely that an efficient actuator could be presently built within the required dimensions. As an example, similar constraints apply to Nafion as well and because commercially available Nafion films are presently $-180 \mu \mathrm{m}$ thick, efficient mechanical design of such ionic conducting polymer film (ICPF) actuators cannot be scaled down below $180 \mu \mathrm{m}$, which is still much too high to be embedded in the suggested minimally invasive recording tip.

Independent micro-actuation on each microelectrode instead of micro-actuation generated by a global field acting on all microelectrodes simultaneously is extremely interesting for two major reasons. First, the recording wire connecting to each microelectrode could be used in alternating phases where electrical actuation signals would be carried toward the electrode in a first phase and then recording signals would be carried from the electrode in a second phase. An inchworm like mechanism supplemented with basic control on direction of motion could provide a mechanism of tracking neuronal activities and based on the intensity of the recorded signals. This in turn would allow the overall recording surface to be lowered by locating each microelectrode tip closer to the source of neuronal activities. Furthermore, the slow motion would be beneficial from a physiological point of view while facilitating the tracking of neurons. Other approaches such as the implantation of neurotrophic growth factors to allow cortical cells to grow toward the electrode tip could be used to complement or to replace the tracking requirement and/or to reduce the electrical impedance required for proper recordings. But it is not clear at the present time if such an approach would be efficient or if the added complexity to integrate such growth factors in the electrode tip would result onto overall larger dimensions.

\section{CONCLUSION}

Making microelectrodes for future brain-machine interfaces less invasive is a priority. Several approaches can be investigated. One proposed approach reduces the overall size of the recording tips during implantation into the brain tissue. Recording tips using bi-stable volumetric shapes have been proposed where the recording surface can be increased once positioned into the cortex. In order to further reduce the overall dimensions of the recording electrodes, one approach proposes the elimination of the insulated part of the microelectrodes. Tracking techniques making use of embedded micro-actuation mechanisms in order to locate the recording tips closer to the neuronal activities can also be used to decrease further the required overall recording surface area.

\section{REFERENCES}

[1] http:/whatis.techtarget.com/definition/0,,sid9_gci521113,00.htm]

[2] J. P. Donohue "Connecting cortex to machines: recent advances in brain interfaces," Nature Neurosciences Suppl., Vol. 5, 1085-1088, Nov. 2002.

[3] J. Wesberg, J. W. Stambaugh, J. D. Kralik, P. D. Beck, M. Laubach, J. K. Chapin, J. Kim, S. J. Biggs, M. A. Srinivasan, and M. A. L. Nicolelis, "Real-time prediction of hand trajectory by ensembles of cortical neurons in primates," Nature, 408, pp. 361-365, 2000.

[4] "Methods for neural ensemble recordings," Edited by Miguel A.L. Nicolelis, CRC Methods in Neurosciences Series, CRC Press, 1999.

[5] S. Martel, N. Hatsopoulos, J. Donoghue, I. Hunter, J. Burgert, J. Malášek, C. Wiseman, and R. Dyer, "Development of a wireless brain implant: the Telemetric Electrode Array System (TEAS) project," Proceedings of the $23^{\text {rd }}$ Annual International Conference of the IEEE Eng. in Medicine and Biology Society, pp. 3594-3597, Oct. 25-28, 2001

[6] T. Fofonoff, C. Wiseman, R. Dyer, J. Malášek, J. Burgert, S. Martel, I. Hunter, N. Hatsopoulos, and J. Donoghue, "Mechanical Assembly of a Microelectrode Array for use in a Wireless Intracortical Recording Device," Proc. of the $2^{\text {nd }}$ Annual Int. IEEE-EMBS Special Topic Conf. on Microtechnologies in Med. \& Biology, pp. 269-272, May 2-4, 2002.

[7] T. Fofonoff, S. Martel, C. Wiseman, R. Dyer, I. Hunter, N. Hatsopoulos, and J. Donoghue, "A Highly Flexible Manufacturing Technique for Microelectrode Array Fabrication," Proc. of the $2^{\text {nd }}$ Joint IEEE-EMBS and BMES Conf., pp. 2107-2108, Oct. 23-26, 2002.

[8] T. Fofonoff, "Brain microelectrode array systems", M.S. Thesis, Mechanical Engineering Department, MIT, 2003.

[9] S. Pellegrino, "CRTS reflectors," Proc. 25th ESA Antenna Workshop on Satellite Antenna Technology, ESTEC, The Netherlands, 2002.

[10] S. Pellegrino, "Deployable membrane reflectors," Proc. 2nd World Engineering Congress, Sarawak, Malaysia, pp. 1-9, 2002.

[11] W. J. Rits, "A multipurpose deployable membrane reflector," ESA Bulletin, 88, pp. 66-71, 1996.

[12] D. S. A. De Focatiis and S. D. Guest, "Deployable membranes designed from folding tree leaves," to be published in the Transactions of the Royal Society of London, Series A, 2002. 